\title{
The Influence of Structural Parameter on the Properties of PCF
}

\author{
Lei $\mathrm{Hu}^{1, \mathrm{a}}$ \\ ${ }^{1}$ Xi'an fanyi university, Xi'an 710105, China \\ aemail: shuhua_y@126.com
}

Keywords: Microstructure Optical Fiber; structural parameter; Chromatic Dispersion; Single-mode

\begin{abstract}
By analyzing the structure and properties of photonic crystal fiber (PCF), a method for calculating cladding-effective-index was proposed. Then, the influence of changing the diameters of fiber core and cladding air holes and the interval of cladding air holes on the single mode property, dispersion, attenuation characteristics and nonlinearity was analyzed. Single mode transmission was . able to be achieved based on the designed PCF in large band width. Anomalous dispersion and the movement of zero dispersion point to the direction of short wavelength were both realized within short wavelength range. Furthermore, the changes of structure parameters in dissimilar layers led to different changes of the properties of PCF.
\end{abstract}

\section{Introduction}

A novel optical fiber with periodic transverse microstructure, namely, photonic crystal fiber (PCF) was presented in the paper. There are air holes in different arrangements on the cross section of PCF. By reasonably designing the unique structure, PCF can have better transmission properties than traditional optical fiber, especially invariant single mode property. That is, only one transmission mode is supported in a large range of band width. Controllable dispersion can also be obtained, including the needed dispersion value, dispersion slope, the position of zero dispersion wavelengths and flat dispersion curve. Furthermore, PCF has excellent optical nonlinearities. The strength of nonlinear effect can be effectively controlled by designing effective fiber core area according to the specific needs ${ }^{[1]}$.

The study on the influence of the changes of structure parameters on the single mode property, dispersion, attenuation characteristics and nonlinearity provides reference for the design of transmission medium and optical devices based on PCF.

\section{Calculation of effective refractive index of PCF}

Scalar approximation theory of the distribution of electromagnetic fields is generally used for calculating the cladding-effective-index.

First, the cladding area of PCF was regarded as a hexagonal two-dimensional photonic crystal with infinitely periodic arrangements and no central defects. Then, circular unit cells with equal area were adopted to substitute the hexagonal ones. Therefore, fiber sections and equivalent unit cells are shown in Reference [2-3].

Since the areas are equal, the equivalent area formula is $R=(\sqrt{3} / 2 \pi)^{1 / 2} \Lambda \approx 0.525 \Lambda$. Where, $\Lambda$ presents the pitch of cladding hole; the radius of circular air column in transverse basic unit cells is $d$ and the outside radius of unit cell is $R$. The field distribution function $\psi$ of transverse basic mode in polar coordinate system needs to satisfy the following scalar wave equation.

$$
\frac{d^{2} \psi}{d \rho^{2}}+\frac{1}{\rho} \frac{d \psi}{d \rho}+\left(k^{2} n_{i}^{2}-\beta^{2}-\frac{m^{2}}{\rho^{2}}\right) \psi=0
$$

For fundamental modes, when $m=0$, the equation can be rewritten as:

$$
\frac{d^{2} \psi}{d \rho^{2}}+\frac{1}{\rho} \frac{d \psi}{d \rho}+\left(\beta^{2}-k^{2} n_{0}^{2}\right) \psi=0, \quad \rho \leq d
$$


$\frac{d^{2} \psi}{d \rho^{2}}+\frac{1}{\rho} \frac{d \psi}{d \rho}+\left(k^{2} n_{\text {silica }}^{2}-\beta^{2}\right) \psi=0, \quad d \leq \rho \leq R$

Where, $n_{0}$ and $n_{\text {silica }}$ are the refractive indexes of air and quartz respectively. When $n_{0}=1$, $n_{\text {silica }}$ can be calculated by Sellmeier equation.

$$
n_{\text {silica }}(\lambda)=\sqrt{1+\sum_{j=1}^{N} \frac{\lambda^{2} B_{j}}{\lambda^{2}-\lambda_{j}^{2}}}
$$

According to the integral order Bessel, its singular form and corresponding recurrence formula, when $\rho=R$, it should meet $d \psi / d \rho=0$ in terms of periodic boundary conditions. When $\rho=d$, that is $\psi$ and $d \psi / d \rho$ should be continuous at the interface of air and quartz, then the expression of $\beta$ can be acquired while the effective refractive index of fiber cladding is $n_{\text {eff }}(\omega)=\beta(\omega) c / \omega$. Therefore, the effective refractive index of cladding of PCF is acquired.

\section{Unique characteristics of PCF}

PCF is a kind of transmission medium comprised of single material (such as $\mathrm{SiO}_{2}$ ). Its unique property is mainly determined by three characteristic parameters including core diameter, the diameter of cladding air holes and the interval of cladding air holes. The unique characteristics of PCF can be obtained by adjusting these three characteristic parameters.

Invariant single mode property Single mode transmission can be realized by PCF in the whole band as long as the design of structure is reasonable. If the ratio of diameter of air holes to the interval of the adjacent holes is less than 0.2, PCF has invariant single mode property with no relation to absolute size, as shown in Figure 1 . The measurement results show that single mode transmission is achieved by PCF in a band range of 337 to $1,550 \mathrm{~nm}$.

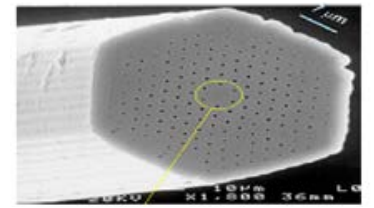

Fig.1 PCF of single-mode

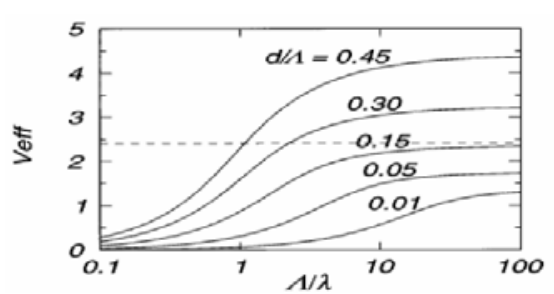

Fig.2 the normalized frequency

The effective normalized frequency V of PCF is expressed as follows ${ }^{[45]}$ :

$$
V_{\text {eff }}=\frac{2 \pi \Lambda}{\lambda} \sqrt{n_{i}^{2}-n_{e f f}^{2}}
$$

Where $\Lambda$ denotes the pitch of cladding holes.

The value of the effective normalized frequency $V$ eff of PCF changes with the variation of normalized wave-number $\Lambda / \lambda$ and relative size of pore diameter $\mathrm{d} / \Lambda$. Veff approaches to a constant value with the increase of $\Lambda / \lambda$ and enlarges with the increase of $d / \Lambda$, as presented in Figure 2 . In short wavelength limit, the value of $V$ eff is not directly associated with $\Lambda$ and $\lambda$, but changes with $d / \Lambda$. In long wavelength range, the approximate limit value of $V$ eff is presented as follows:

$$
V_{e f f}=k \Lambda F^{1 / 2} \sqrt{n_{0}^{2}-n_{a}^{2}}
$$

Where, $n_{a}$ is air refractive index and $F$ is the proportion of filled air, namely, the area of air in cladding of PCF. $F=(\pi / 2 \sqrt{3}) \times(d / \Lambda)^{2}$ 。

At the same time, $\mathrm{V}$ eff reduces with the increase of wavelength in the direction of long wavelength. According to the light-transmitting principles of common fiber, when limit value is less than 2.405, single mode transmission in the whole band is able to be achieved.

Controllable dispersion The transverse refractive index of PCF and its dispersion are highly 
adjustable. The dispersion of PCF was numerically stimulated using multi-stage method, hence the relation between dispersion value and wavelength $\lambda$, pore diameter $d$, hole interval $\Lambda$ and filling rate can be acquired ${ }^{[6]}$.

Figure 3 illustrates the variation of dispersion $D$ of PCF with the hole interval $\Lambda$, and the influence of the changes of the hole interval on dispersion can be known.
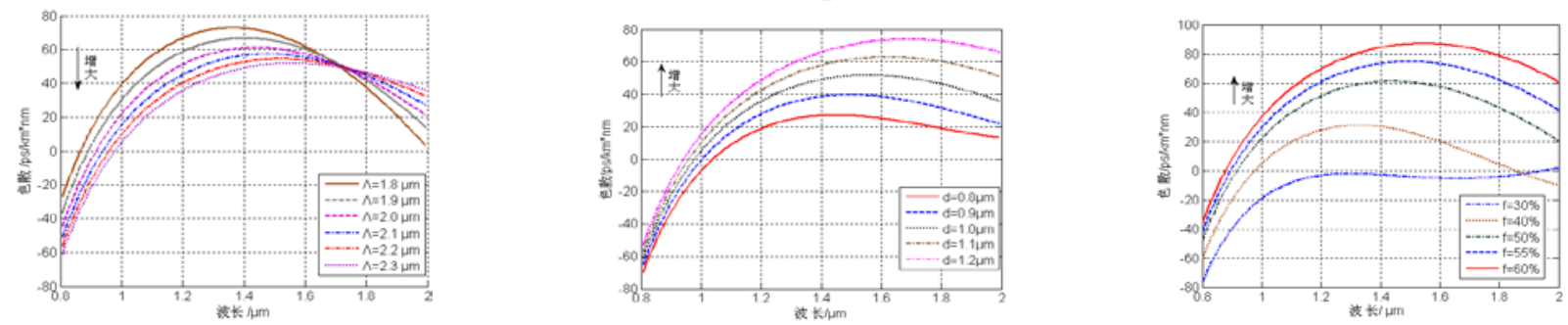

Fig. 3 the dispersion curve of different $\Lambda$ with vary $\lambda$ Fig.4 the dispersion curve of different $\mathrm{d}$ with vary $\lambda$ Fig.5 the dispersion curve of different $\mathrm{f}$ with vary $\lambda$

The figure shows that with constant diameter of air holes, dispersion $D$ is distinctly changed by adjusting the hole interval $\Lambda$. All the curves intersect at $1.63 \mu \mathrm{m}$. Dispersion $D$ reduces with the increase of $\Lambda$ in the direction from $1.63 \mu \mathrm{m}$ to short wavelength; while it increases with the increase of $\Lambda$ in the direction from $1.63 \mu \mathrm{m}$ to long wavelength. There are two intersection points of each curve and zero dispersion line, and they move to the direction of long wavelength with the gradual increase of $\Lambda$ with gradually broad distance and flat curve.

Figure 4 presents the variation of dispersion $D$ of PCF with the changes of pore diameter $d$.

Figure 5 illustrates the variation of dispersion $D$ of PCF with the changes of air filling rate $f$.

Little attenuation characteristics Since the attenuation of PCF depends on its structure, the working attenuation of reasonably designed PCF is small, which can be proved by measurements. The attenuation of PCF was measured by insertion method using white light sources and a spectrum analyzer. The length of the tested fiber is $2.5 \mathrm{~km}$ and the obtained fiber attenuation is demonstrated in Figure 6 (left). To detect the inhomogeneity including scattering points of the fiber, an optical time domain reflectometry (OTDR) with a working wave of $1,550 \mathrm{~nm}$ and a pulse width of $10 \mathrm{~ns}$ was inserted along the direction of fiber length. The solid lines in Figure 6 (right) presents the obtained curve. The attenuation measured by insertion method at $1,550 \mathrm{~nm}$ is consistent with that by OTDR.
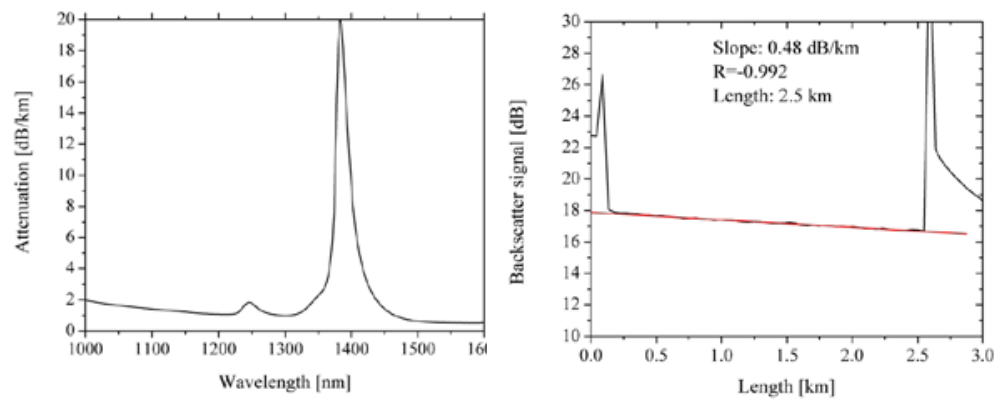

Fig.6 attenuation curve and the measured curve of OTDR in 1550nm

In view of the data in Figure 6, owing to the existence of $\mathrm{OH}-$, when the concentration of $\mathrm{OH}$ reached $0.4 \mathrm{ppm}$, there was an attenuation peak nearly $20 \mathrm{~dB} / \mathrm{km}$ at $1,380 \mathrm{~nm}$. While at 1,550 $\mathrm{nm}$, the value of attenuation was $0.15 \mathrm{~dB} / \mathrm{km}$. These measurement results were agreed to the expected values. Rayleigh scattering coefficient was approximate $1 \mathrm{~dB} /\left(\mathrm{km} \cdot \mu \mathrm{m}^{4}\right)$ and the scattering value at $1,550 \mathrm{~nm}$ was $0.18 \mathrm{~dB} / \mathrm{km}$. While the attenuation value of $0.16 \mathrm{~dB} / \mathrm{km}$ was caused by the absorption result from impure materials and defects. Taking $\mathrm{d} / \Lambda$ and $\Lambda$ into consideration, the effective area of fundamental mode was $130 \mu^{2}$ and the Gaussian mode field diameter (MFD) was $13 \mu \mathrm{m}$. These indicate that PFC has large effective area and low loss ${ }^{[7]}$.

A Excellent nonlinearity The nonlinear effect of PCF is determined by the product of nonlinear coefficient and the optical power of fiber. The nonlinear coefficient $r$ of PCF is expressed as 
follows.

$$
r=k \sum_{i} n_{2}^{i} / A_{\text {eff }}^{i}=k n_{2}^{e f f} / A_{\text {core }}
$$

Where $n_{2}^{\text {eff }}$ is the effective refractive index of fiber cladding and $A_{\text {eff }}$ presents the effective mode area. From formula (7) we can know that the nonlinear coefficient is inversely proportional to parameter $A_{\text {eff }}$, while $A_{\text {eff }}$ depends on the structure parameter of fiber ${ }^{[8]}$.

When $\Lambda$ was set as $\Lambda=2.3 \mu \mathrm{m}$, the larger the pore diameter $d$, the smaller the effective mode area $A_{\text {eff }}$ was, as illustrated in Figure 7.

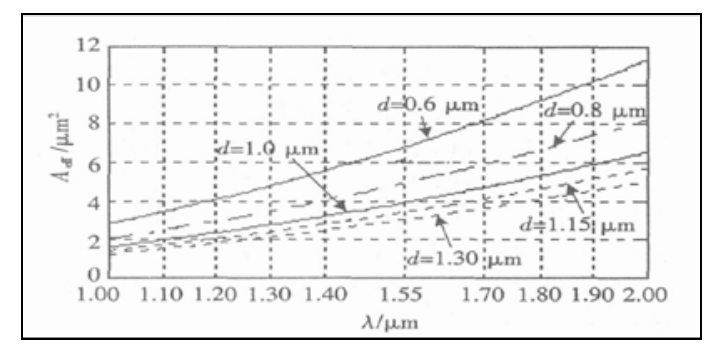

Fig.7 the influence of different d to effective mode area

It can be seen from Figure 7 that when $d / \Lambda$ was fixed, the effective mode area $A_{\text {eff }}$ increased with the increase of wavelength. When wavelength was fixed, the effective mode area $A_{\text {eff }}$ reduced with the increase of $d / \Lambda$ value. When $d / \Lambda$ was larger than a certain value, $A_{\text {eff }}$ was less than $6 \mu \mathrm{m}^{2}$ in a large band range. The reduction of fiber core diameter can improve the restriction of light, hence reducing the loss of light. In the condition, the light power density in unit area of core section can be increased, which enables light transmitted in optical fiber to generate various nonlinearities.

\section{Conclusions}

By changing the structure parameter, the influence of structure on properties of PCF was analyzed. The changes of diameters of fiber core and cladding air holes and the interval of cladding air holes realized the single mode transmission in large band width. Furthermore, anomalous dispersion was found in short wavelength range and zero dispersion point moved to the direction of short wavelength. Besides, nonlinear effects were realized in the minimization of the working attenuation. All these properties are not exhibited by traditional optical fibers.

\section{References}

[1] Russell P St J, Knight J C, Birlcs T A, et al. (2000)Recent progress in photonic crystal fibres. Optical Fiber Communication Conference, 3: 98 100.

[2] Yinian Zhu, Ping Shum, Hin-Joo Chong, et al. (2003)Strong resonance and a highly compact long period grating in a large-mode-area photonic crystal fiber. Optics Express, 11(16):1900-1995.

[3] Jacob Lasri, Preetpaul Devgan, Renyong Tang, et al.(2003)A microstructure-fiber-based $10-\mathrm{GHz}$ synchronized tunable optical parametric oscillator in the 1550-nm regime. IEEE Photonics Technology Letters,15(8): 1058-1060.

[4] T.A. Birks, J.C. Knight, P.St.J. Russell.(1997) Endlessly single-mode photonic crystal fiber. Optics Letters, 22(13):961 963.

[5] Feng Chaoyin,Wang Jixian.(2014)Analysis of the properties of a novel high birefringence photonic crystal fiber. Study on Optical Communications, 02(1): 41-44.

[6] Li Honglei, Lou Shuqin, Guo Tieying, et al. (2010)Low loss fusion splicing of germanium doped core photonic crystal fiber and standard single mode fiber. Chinese Journal of Lasers, 
37(6): 1589-1593.

[7] G.Genty,H.Ludvigsen.(2004)Meassssurement of anomalous dispersion in microstructured fiber using spectral modulation.Optical Express, 12(5):929-934.

[8] Philip St.J. Russell.(2006)Photonic-Crystal Fibers. Journal Of Lightwave Technology. 24(12) . 